\title{
MicroRNA-mediated regulation of target genes in several brain regions is correlated to both microRNA-targeting-specific promoter methylation and differential microRNA expression
}

Y-h Taguchi

Correspondence: tag@granular.com Department of Physics, Chuo University, Tokyo 112-8551, Japan

\begin{abstract}
Background: Public domain databases nowadays provide multiple layers of genome-wide data e.g., promoter methylation, mRNA expression, and miRNA expression and should enable integrative modeling of the mechanisms of regulation of gene expression. However, researches along this line were not frequently executed.

Results: Here, the public domain dataset of mRNA expression, microRNA (miRNA) expression and promoter methylation patterns in four regions, the frontal cortex, temporal cortex, pons and cerebellum, of human brain were sourced from the National Center for Biotechnology Informations gene expression omnibus, and reanalyzed computationally. A large number of miRNA-mediated regulation of target genes and miRNA-targeting-specific promoter methylation were identified in the six pairwise comparisons among the four brain regions. The miRNA-mediated regulation of target genes was found to be highly correlated with one or both of miRNA-targeting-specific promoter methylation and differential miRNA expression. Genes enriched for Kyoto Encyclopedia of Genes and Genomes (KEGG) pathways that were related to brain function and/or development were found among the target genes of miRNAs whose differential expression patterns were highly correlated with the miRNA-mediated regulation of their target genes.

Conclusions: The combinatorial analysis of miRNA-mediated regulation of target genes, miRNA-targeting-specific promoter methylation and differential miRNA expression can help reveal the brain region-specific contributions of miRNAs to brain function and development.
\end{abstract}

Keywords: MicroRNA, Target gene regulation, Brain regions, Promoter methylation, Pathway analysis

\section{Background}

miRNAs are short non-coding RNAs that are believed to suppress target gene expression through the binding of miRNA "seed" regions to complementary sequences of 3' untranslated regions (UTR) of target genes [1]. miRNAs are generally assumed to regulate cellular processes related to animal development [2] and cellular differentiation, and have been

C 2013 Taguchi; licensee BioMed Central Ltd. This is an Open Access article distributed under the terms of the Creative Commons Attribution License (http://creativecommons.org/licenses/by/2.0), which permits unrestricted use, distribution, and reproduction in any medium, provided the original work is properly cited. 
implicated in several diseases, including cancer. Thus, miRNAs have been put forth as candidates for tumor suppression [3] and cancer biomarkers [4]. miRNAs are also known to be involved in reprogramming [5]. As such, miRNAs are considered to play critical roles in a wide range of biological processes.

Recently, miRNA expression in the brain has attracted the interest of many researchers [6-9]. Although there are extensive researches about miRNA regulation of target genes [6,7], it is generally believed that the expression of many genes is regulated by miRNAs indirectly [10]. In this sense, in order to understand miRNA regulation of gene expression in brain regions, it is also important to understand the mechanisms by which such regulation occurs.

Together with miRNAs, transcription factors (TFs) bind to promoter regions and cooperatively regulate miRNA target genes [11-15]. TFs form a protein complex that binds to gene promoters during the initiation of transcription. Since there are many TFs known to regulate biological processes in regions of the brain [16-18], it is natural to investigate the combinatorial effects of TFs and miRNA gene regulation in the brain $[19,20]$. In contrast to what is known about cooperative regulation by miRNA and TFs, investigations of gene coregulation mediated by both miRNA and promoter methylation are limited; however, siRNA-induced promoter methylation in CpG islands has been reported [21-24]. Promoter methylation is generally thought to suppress gene expression [25]. Suppression of gene expression by promoter methylation is often important. For example, aberrant promoter methylation is often related to cancers [26,27]. Promoter methylation also plays critical roles in reprogramming [28].

Despite the known importance of promoter methylation, the relationship between promoter methylation and miRNA-mediated gene regulation has received little attention. However, it was recently shown that promoters of genes not targeted by miRNAs have higher levels of methylation [29]. We recently found that miRNA-targeting-specific promoter methylation takes place in many cell lines [30,31]. miRNA-targeting-specific promoter methylation refers to the association between 3 BUTR miRNA targetting and promoter methylation levels for a given gene.

In this paper, we report that miRNA-targeting-specific promoter methylaion also exists between distinct brain-regions in a brain-region specific manner. Considered brain regions are frontal cortex, temporal cortex, pons, and cerebellum [32]. The frontal cortex is located at the front of the head in human. It is considered to be the hub of most higher functions and understanding, and is believed to govern most behavioral traits, motor skills, and problem solving tactics [33]. The temporal cortex is located in the lower right and left regions of the brain, and is involved in hearing, understanding languages, face recognition, and certain memory functions [34]. The cerebellum is located in the lower region at the back of the brain, and is central to motion control [35]. Finally, the pons is located in the center of these three regions and mediates information transfer between several other brain regions, including the cortex and cerebellum [32]. Given the diverse functions of these brain regions, I hypothesized that miRNA-targeting-specific promoter methylation would occur in a region-specific manner. Not only did I determine that patterns of miRNA regulation were indeed brain-region specific, I also revealed that some miRNA regulation of target genes turned out to be controlled by not only differential miRNA expression itself but also miRNA-targeting-specific promoter methylaion. In addition, target genes of miRNAs whose regulation was significantly correlated to 
differential miRNA expression were also found to be enriched for brain-region-specific functions and related KEGG pathways.

\section{Methods}

Patterns of miRNA and mRNA expression and promoter methylation

Datasets used in this study were downloaded from Gene Expression Omnibus (GEO) under GEO ID GSE15745. These included miRNA and mRNA expression, and promoter methylation data from four distinct brain regions (frontal cortex, temporal cortex, pons and cerebellum) in 150 human subjects [36], which had been analyzed in detail in connection with genomic variants, such as single nucleotide polymorphisms and copy number variants; however, miRNA expression had not been analyzed previously [36]. Thus, in total, 600 tissue samples were included. Processed signals were used without any further normalization. For more details about data processing and analysis, see the Supplementary Document (see Additional file 1).

\section{Results and discussion}

In this section, I will discuss the mutual relationships between miRNA-related features and their biological meaning.

Mutual relationships between miRNA-mediated regulation of genes, miRNA-targeting-specific promoter methylation, and differential miRNA expression

I investigated miRNA-mediated gene regulation and miRNA-targeting-specific promoter methylation in the frontal cortex, temporal cortex, pons, and cerebellum of the human brain, based on the $P$-values, $P_{m j,<}^{\ell \ell}$ s or $P_{m j,>}^{\ell \ell^{\prime}}$ s, which were used to estimate miRNAmediated gene regulation and miRNA-targeting-specific promoter methylation. Figure 1 illustrates the results of this analysis. It is clear that target genes of a substantial number of miRNAs are up/downregulated between these four brain regions. It is also evident that some miRNA target genes are differentially methylated between these four brain regions. This strongly suggests that both miRNA-mediated gene regulation and miRNA-targetingspecific promoter methylation play critical roles in the development and function of these four brain regions. For example, from the miRNA-centric point of view (Figure 1), compared to the other three brain regions investigated, the pons has more genes with hypermethylated promoters and lower expression levels, although these characteristics are not always associated. This observation is consistent with the general belief that the hypermethylation of promoters is associated with reduced expression. This also signifies that mRNA expression in the pons is distinct from the other three brain regions.

\section{Mutual relationships between miRNA-mediated regulation of target genes and miRNA-targeting-specific promoter methylation}

In order to understand the mutual relationship between miRNA-mediated gene regulation and miRNA-targeting-specific promoter methylation, I computed the correlation coefficient of the mean rank of $P$-values, $\rho_{\ell \ell^{\prime}}^{m R N A, M e t h y l}$, for six pairwise comparisons between the frontal cortex, temporal cortex, pons, and cerebellum (see Figure 1). Here, the means were taken over all samples in each brain region. Excluding a single pairwise comparison between the cerebellum and pons, correlation coefficients for the remaining five comparisons varied between 0.25 and 0.51 . These values were considered to be 


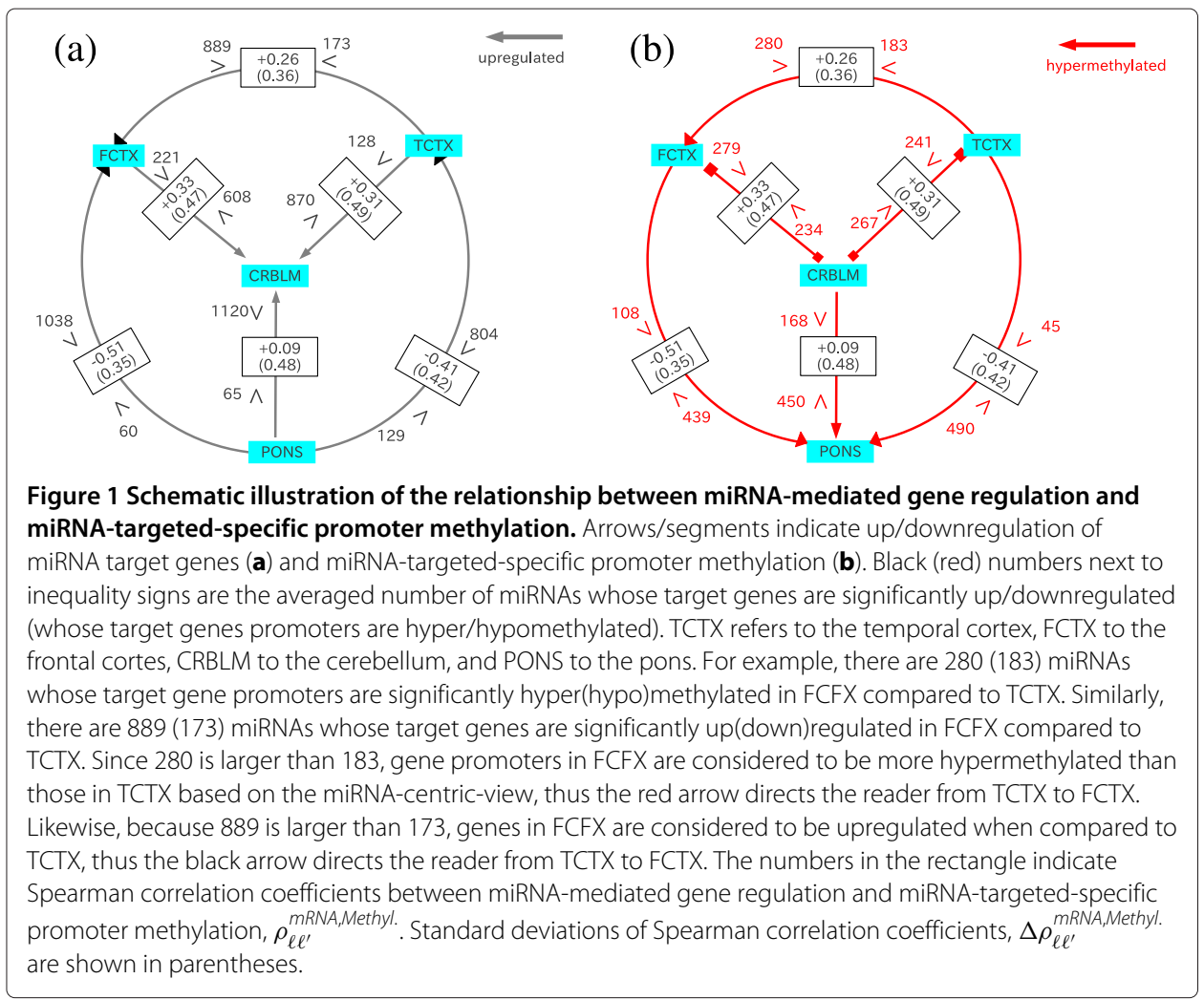

sufficiently large taking into account the fact that the number of $P$-values in a given brain region is as large as $M$, the number of miRNAs comsidered. The $P$-values of each correlation coefficient are less than $2.2 \times 10^{-16}$. This means, the correlation between miRNA-mediated gene regulation and miRNA-targeting-specific promoter methylation is highly significant independent of pairs of brain regions. The smallest correlation coefficients were observed in the cerebellum and pons. Although the correlation coefficient was large in aggregate (0.09), individual $P$-value was as small as $4 \times 10^{-5}$, which is highly significant.

In order to confirm the correlation between miRNA-mediated gene regulation and miRNA-targeting-specific promoter methylation, the root mean squared averages of the correlation coefficients in each sample, $\Delta \rho_{\ell \ell^{\prime}}^{m R N A, M e t h y l}$, were also computed. Excluding pairwise comparisons for the frontal cortex and pons for which the absolute value of $\rho_{\ell \ell^{\prime}}^{m R N A, M e t h y l .}$ was the maximum, $\Delta \rho_{\ell \ell^{\prime}}^{m R N A, M e t h y l .}$ was larger than the absolute value of $\rho_{\ell \ell^{\prime}}^{m R N A, M e t h y l .}$. This signifies that the correlation coefficients within each sample were not small, but that when averaged over all samples, the value was seemingly small because of the occurrence of both positive and negative correlations with equal probabilities. Thus, I conclude that miRNA-mediated regulation and miRNA-targeting-specific promoter methylation are significantly correlated. Worth noting is that the signs of correlation coefficients, $\rho_{\ell \ell^{\prime}}^{m R N A, M e t h y l}$, are neither definitively positive nor negative. One may think that they should be positive, as both promoter methylation and miRNA targeting should suppress gene expression. However, because genes targeted by miRNAs are expected to be downregulated (upregulated) only when miRNA itself is upregulated (downregulated), there is no reason to expect that the correlation coefficients between miRNA-mediated 
gene regulation and miRNA-targeting-specific promoter methylation should always take positive or negative values.

Relationships between miRNA-mediated regulation of target genes, miRNA-targeting-specific promoter methylation and differential miRNA expression

In order to determine the relationship between miRNA-mediated gene regulation, $P_{m j,<}^{\ell \ell^{\prime}}$ or $P_{m j,>}^{\ell \ell^{\prime}}$, and differential expression of miRNA, $\log \left(\frac{x_{m j \ell}}{x_{m i \ell^{\prime}}}\right)$, the correlation coefficients were computed. However, these correlation coefficients were too small to be significant (not shown here). This seemingly contradicts the observed correlation between miRNAmediated gene regulation and miRNA-targeted-specific promoter methylation.

Thus, in order to resolve this apparent discrepancy, I employed multivariate regression models between miRNA-mediated gene regulation, miRNA-targeting-specific promoter methylation, and differential miRNA expression, also considering both sample gender and age (see Methods). In contrast to the above discrepancy, depending upon the miRNA considered, I identified significant correlations between only selected variables that were included in the regression model. In other words, I found that all of the variables were not always correlated, but were instead selectively correlated. In order to quantize these correlations, for each miRNA, I picked out the combinations of variables that were significantly correlated (see Methods). Table 1 lists the miRNAs selected for each pair of brain regions based on the criterion described in the subsection, "The selection of miRNAs that significantly regulate target genes based on multiple regression" in Supplementary Document (see Additional file 1), i.e., miRNAs whose differential expression is significantly correlated to miRNA-mediated gene regulation. To our knowledge, this is the first time that miRNA gene regulation has been shown to be mediated by both differential miRNA expression and miRNA-targeting-specific promoter methylation.

\section{Biological meanings of findings}

As can be seen in Table 1, miRNAs selected for each pair of brain regions are not unique, but rather divergent. Some of the listed miRNAs were previously reported to be important in specific brain regions. For example, Yao et al recently investigated miRNA expression in the rat cerebral cortex during brain development [37]. Many of the top 20 most highly expressed miRNAs identified by Yao et al at each of eight different developmental stages, ranging from early developmental stages to late post natal stages, were also significant in our dataset (rno-let-7b, 7e, 7i, rno-miR-181b, 99a/b, 9, 125b-5p, and 191). Yao et al also emphasized the importance of miR-137, the ortholog of the human miRNA, hsa-miR-137; this miRNA was found to be significant twice in our analysis, compared to the most of other miRNAs which were only identified as significant once. In addition, many of the miRNAs listed in Table 1 have also been previously implicated in brain diseases, including Alzheimers disease (AD), Parkinsons disease (PD), Huntingtons disease (HD), and various other neurodegenerative disorders [38,39]. This overlap lends support to the utility of our method for identifying miRNAs with potential functional relevance in the brain. Although there have been other investigations of brain miRNA expression, to our knowledge, I am the first to interrogate miRNA expression data across multiple brain regions. 
Table 1 miRNAs that significantly regulate target genes

\begin{tabular}{|c|c|c|c|c|c|}
\hline \multicolumn{2}{|c|}{ CRBLM vs FCTX } & \multicolumn{2}{|c|}{ CRBLM vs PONS } & \multicolumn{2}{|c|}{ CRBLM vs TCTX } \\
\hline Reciprocal & Nonreciprocal & Reciprocal & Nonreciprocal & Reciprocal & Nonreciprocal \\
\hline hsa-miR-181c-5p & hsa-miR-200a-5p & hsa-miR-20a-5p & hsa-let-7b-5p & hsa-miR-210 & hsa-miR-99a-5p \\
\hline hsa-miR-135a-5p & hsa-miR-381 & hsa-miR-23a-3p & hsa-let-7e-5p & & hsa-miR-191-5p \\
\hline hsa-miR-137 * & hsa-miR-202-3p * & hsa-miR-148a-3p & hsa-miR-197-3p & & hsa-miR-99b-5p * \\
\hline hsa-miR-363-3p & hsa-miR-561-3p & hsa-miR-10a-5p & hsa-miR-181b-5p & & hsa-miR-617 \\
\hline hsa-miR-369-3p & hsa-miR-568 & hsa-miR-221-3p & hsa-let-7i-5p & & \\
\hline hsa-miR-487a * & hsa-miR-618 & hsa-miR-223-3p & hsa-miR-9-5p & \multicolumn{2}{|c|}{ FCFX vs PONS } \\
\hline hsa-miR-514a-3p & hsa-miR-630 * & hsa-miR-1 & hsa-miR-126-3p & hsa-miR-365a-3p & hsa-miR-302d-3p \\
\hline hsa-miR-553 & & hsa-miR-133a & hsa-miR-134 & hsa-miR-378a-5p & hsa-miR-432-5p \\
\hline hsa-miR-554 & & hsa-miR-137 * & hsa-miR-154-3p & & hsa-miR-595 \\
\hline hsa-miR-655 & & hsa-miR-146a-5p & hsa-miR-299-5p & & \\
\hline \multirow[t]{16}{*}{ hsa-miR-421 } & & hsa-miR-452-5p & hsa-miR-99b-5p * & \multicolumn{2}{|c|}{ FCTX vs TCTX } \\
\hline & & hsa-miR-484 & hsa-miR-377-3p & has-miR-373-3p & hsa-miR-24-3p \\
\hline & & hsa-miR-511 & hsa-miR-383 & & hsa-miR-485-5p \\
\hline & & hsa-miR-515-5p & hsa-miR-431-5p & & hsa-miR-766-3p \\
\hline & & hsa-miR-571 & hsa-miR-329 & & \\
\hline & & hsa-miR-549 & hsa-miR-485-3p & \multicolumn{2}{|c|}{ PONS VS TCTX } \\
\hline & & & hsa-miR-487a * & hsa-miR-9-3p & hsa-miR-222-3p \\
\hline & & & hsa-miR-202-3p * & hsa-miR-302a-3p & hsa-miR-125b-5p \\
\hline & & & hsa-miR-432-3p & hsa-miR-410 & hsa-miR-328 \\
\hline & & & hsa-miR-495 & hsa-miR-487b & hsa-miR-581 \\
\hline & & & hsa-miR-504 & hsa-miR-630 * & hsa-miR-661 \\
\hline & & & hsa-miR-505-3p & & \\
\hline & & & hsa-miR-563 & & \\
\hline & & & hsa-miR-578 & & \\
\hline & & & hsa-miR-630 * & & \\
\hline & & & hsa-miR-668 & & \\
\hline
\end{tabular}


In order to better understand the biological functions of the miRNA targets identified in our analysis, I employed pathway analysis (Table 2), which has been shown previously to be effective for miRNA target genes (e.g., [40,41]). For this purpose, I used DIANAmirPath [42], which is a web tool developed for KEGG pathway enrichment analysis of miRNA target genes.

Compared to the variation observed in the miRNAs listed in Table 1, KEGG pathways for miRNA targets (Table 2) are highly universal and biologically meaningful as shiwn in the followings. For example, Paul et al [43] measured and analyzed transcritpomes in the mouse cerebellum. Cells were classified into Purkinje cells (PCs) at postnatal days 3, 7, 14, 21, 28, 35, and 56 (P3, P7, P14, P21, P28, P35, and P56), and the mixture of Stellate/Basket cells (StCs/BKCs) at P14, P21, P28, P35, and P56. They conducted pathway enrichment analysis using KEGG pathways based on developmental gene expression of PCs and S/BCs. From this, they found that many pathways were enriched at several different time points. In their data, upregulated genes identified between P3-PCs and P7-PCs were enriched for pathways such as "axon guidance", "regulation of actin cytoskeleton", "gap junction", and "tight junctions", implicating roles for these genes in the early stages of circuit integration by PCs. These changes are accompanied by an upregulation of other pathways such as insulin, TGF- $\beta$, Hedgehog, and Wnt signaling, which are important for axon guidance. The upregulation of GnRH signaling, which is known to have a modulatory effect on cerebellar neurons and P53 signaling, and is important for PC survival was also observed during this time.

In P14-PCs, Paul et al also reported that pathways related to "long-term potentiation", "long-term depression", "JAK/STAT", "VEGF", and "mTOR signaling" were elevated, which correlate to the development of parallel fiber synapses. Between P28 and P56, the upregulation of pathways related to "CAMs", "chondroitin sulfate biosynthesis", "focal adhesion", "cytokine receptor interaction", and "extracellular matrix receptor interaction" (ECM-interaction) correlate with the maturation and stabilization of PC connectivity. In S/BCs a number of similar pathways are also activated. "axon guidance", "tight junction", "adherens junction", "insulin signaling", "ErbB", and "spliceosome" pathways were upregulated in P14S/BCs, reflecting delayed axogenesis of BskC and StC after they enter the ML during the second postnatal week. However, between P28 and P35, similar to PC cells, pathways of "ECM-receptor interaction", "CAMs", "cytokine receptor interaction", "neuroactive ligand receptor interactions" and "regulation of cytoskeleton" were activated. These listed pathways largely overlap those listed in Table 2. Although Paul et al mainly investigated the cerebellum, I studied the cerebellum, as well as the pons, and frontal and temporal cortex; thus, I investigated previous studies related to individual pathways listed in Table 2 one by one.

\section{Pathways directly related to brain/nervous system}

Some pathways listed in Table 2 are obviously related to the brain and/or nervous system. For example, "axon guidance" is definitely included in brain development. "Glioma" is a brain tumor and the "neurotrophin signaling pathway" is related to neural systems. Enrichments in these three pathways further supports the notion that the genes I have identified are indeed relevant to brain function and development. For additional discussion of other selected pathways, see the Supplementary Document (see Additional file 1). 
Table 2 miRNA target genes KEGG pathway enrichment

\begin{tabular}{|c|c|c|c|c|c|c|c|c|c|c|c|c|c|}
\hline & \multirow[b]{2}{*}{ KEGG pathways } & \multicolumn{2}{|c|}{$\begin{array}{l}\text { CRBLM } \\
\text { vs } \\
\text { FCTX }\end{array}$} & \multicolumn{2}{|c|}{$\begin{array}{l}\text { CRBLM } \\
\text { vs } \\
\text { PONS }\end{array}$} & \multicolumn{2}{|c|}{$\begin{array}{l}\text { CRBLM } \\
\text { vs } \\
\text { TCTX }\end{array}$} & \multicolumn{2}{|c|}{$\begin{array}{c}\text { FCTX } \\
\text { vs } \\
\text { PONS }\end{array}$} & \multicolumn{2}{|c|}{$\begin{array}{c}\text { FCTX } \\
\text { vs } \\
\text { TCTX }\end{array}$} & \multicolumn{2}{|c|}{$\begin{array}{l}\text { PONS } \\
\text { vs } \\
\text { TCTX }\end{array}$} \\
\hline & & $\mathbf{R}$ & $\mathbf{N}$ & $\mathbf{R}$ & $\mathbf{N}$ & $\mathbf{R}$ & $\mathrm{N}$ & $\mathbf{R}$ & $\mathbf{N}$ & $\mathbf{R}$ & $\mathbf{N}$ & $\mathbf{R}$ & $\mathrm{N}$ \\
\hline 1 & TGF- $\beta$ signaling pathway & $\bigcirc$ & $\bigcirc$ & 0 & $\bigcirc$ & & & & $\bigcirc$ & & & $\mathrm{O}$ & \\
\hline 2 & Glioma * & $\bigcirc$ & $\bigcirc$ & 0 & $\bigcirc$ & $\bigcirc$ & & & $\bigcirc$ & & & & \\
\hline 3 & MAPK signaling pathway & $\bigcirc$ & $\bigcirc$ & 0 & $\bigcirc$ & & & & & & $\bigcirc$ & & \\
\hline 4 & Axon guidance $*$ & $\bigcirc$ & $\bigcirc$ & $\bigcirc$ & $\bigcirc$ & & & & & & & & \\
\hline 5 & Phosphatidylinositol signaling system & $\bigcirc$ & $\bigcirc$ & & $\bigcirc$ & & & & & & & & \\
\hline 6 & mTOR signaling pathway & $\bigcirc$ & $\bigcirc$ & $\bigcirc$ & & & & & & & & & \\
\hline 7 & Adipocytokine signaling pathway & $\bigcirc$ & 0 & & $\bigcirc$ & & 0 & & & & & & \\
\hline 8 & Pancreatic cancer & $\bigcirc$ & $\bigcirc$ & & $\bigcirc$ & & & & $\bigcirc$ & & & & \\
\hline 9 & Endocytosis & $\bigcirc$ & $\bigcirc$ & $\bigcirc$ & $\bigcirc$ & & & & & & & $\bigcirc$ & \\
\hline 10 & Focal adhesion & $\bigcirc$ & $\bigcirc$ & $\bigcirc$ & $\bigcirc$ & & & & & & & $\bigcirc$ & \\
\hline 11 & Insulin signaling pathway & $\bigcirc$ & $\bigcirc$ & $\bigcirc$ & $\bigcirc$ & & & & & & & & \\
\hline 12 & Neurotrophin signaling pathway $*$ & $\bigcirc$ & 0 & 0 & $\bigcirc$ & & & & & & & & \\
\hline 13 & Colorectal cancer & $\bigcirc$ & $\bigcirc$ & $\bigcirc$ & $\bigcirc$ & & & & & & & & \\
\hline 14 & Arrhythmogenic right ventricular cardiomyopathy (ARVC) & $\bigcirc$ & $\bigcirc$ & $\bigcirc$ & $\bigcirc$ & & & & & & $\bigcirc$ & & \\
\hline 15 & Wnt signaling pathway & $\bigcirc$ & $\bigcirc$ & $\bigcirc$ & $\bigcirc$ & & $\bigcirc$ & & & & $\bigcirc$ & & \\
\hline 16 & Non-small cell lung cancer & $\bigcirc$ & $\bigcirc$ & & & & & & & & & & \\
\hline 17 & Adherens junction & $\bigcirc$ & $\bigcirc$ & $\bigcirc$ & $\bigcirc$ & & & & & & & O & \\
\hline 18 & ErbB signaling pathway & $\bigcirc$ & $\bigcirc$ & 0 & $\bigcirc$ & & & & & & & & \\
\hline 19 & Pathways in cancer & $\bigcirc$ & $\bigcirc$ & $\bigcirc$ & $\bigcirc$ & & & & $\bigcirc$ & & & $\bigcirc$ & \\
\hline 20 & Glycosaminoglycan biosynthesis - heparan sulfate & $\bigcirc$ & $\bigcirc$ & $\bigcirc$ & $\bigcirc$ & & & & $\bigcirc$ & $\bigcirc$ & & & \\
\hline 21 & Type II diabetes mellitus & $\bigcirc$ & $\bigcirc$ & & & & & & & & & & \\
\hline 22 & Melanoma & $\bigcirc$ & $\bigcirc$ & & $\bigcirc$ & & & & $\bigcirc$ & & & & \\
\hline 23 & Renal cell carcinoma & $\bigcirc$ & $\bigcirc$ & $\bigcirc$ & $\bigcirc$ & & & & & & & & \\
\hline 24 & Inositol phosphate metabolism & $\bigcirc$ & $\bigcirc$ & & & & & & & & & & \\
\hline 25 & Chronic myeloid leukemia & $\bigcirc$ & $\bigcirc$ & $\bigcirc$ & $\bigcirc$ & & & & $\bigcirc$ & & & & \\
\hline 26 & T cell receptor signaling pathway & $\bigcirc$ & $\bigcirc$ & & & & & & & & & & \\
\hline 27 & Small cell lung cancer & $\bigcirc$ & $\bigcirc$ & $\bigcirc$ & & & & & & & & & \\
\hline 28 & Fc gamma R-mediated phagocytosis & $\bigcirc$ & $\bigcirc$ & & $\bigcirc$ & & & & & & & & \\
\hline 29 & Prostate cancer & $\bigcirc$ & $\bigcirc$ & $\bigcirc$ & & & & & & & & & \\
\hline 30 & Salivary secretion & $\bigcirc$ & $\bigcirc$ & & & & & & & & & & \\
\hline 31 & Osteoclast differentiation & $\bigcirc$ & $\bigcirc$ & & & & & & & & & & \\
\hline 32 & Regulation of actin cytoskeleton & $\bigcirc$ & $\bigcirc$ & 0 & $\bigcirc$ & & & & & & & & \\
\hline 33 & Endocrine and other factor-regulated calcium reabsorption & $\bigcirc$ & $\bigcirc$ & & & & & & & & & & \\
\hline
\end{tabular}


Table 2 miRNA target genes KEGG pathway enrichment (continued)...

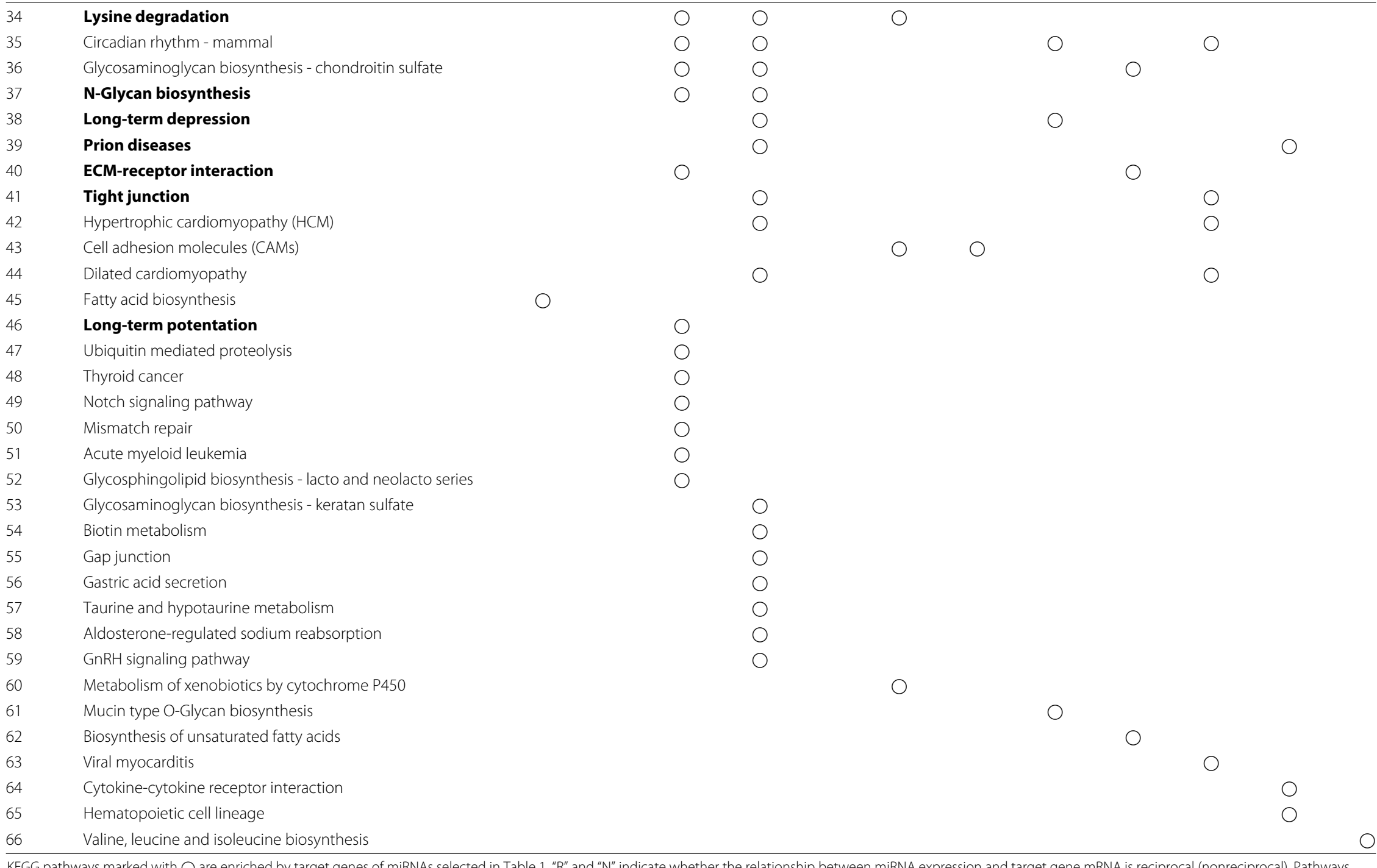

KEGG pathways marked with are enriched by target genes of miRNAs selected in Table 1 " $\mathrm{R}$ " and " $\mathrm{N}$ " indicate whether the relationship between miRNA expression and target gene mRNA is reciprocal (nonreciprocal). Pathways asterisked and bold faced are directly related to brain and neurons, respectively, and discussed in detail in the Supplementary Document (see Additional file 1). 


\section{Other notable observations}

For further discussion of the divergence of miRNAs selected vs. the uniformity of pathways selected, positive vs. negative correlations between miRNA expression and target gene expression, and possible biological explanations underlying coregulation by both miRNA and promoter methylation, see the Supplementary Document (see Additional file 1).

\section{Conclusion}

In this paper, I demonstrated possible miRNA coreguation of target genes in brain regions by analyzing both differential miRNA expression and miRNA-targeting-specific promoter methylation. Selected miRNAs were enriched in brain-related KEGG pathways. Because this was simply descriptive and no mechanisms responsible for the cooperative regulation described above were presented, experiment-based follow up studies will be necessary to validate our findings.

\section{Additional file}

Additional file 1: Supplementary Document: Supplementary information not included in the paper.

Competing interests

The author declares that they have no competing interests.

Acknowledgements

This research was supported by KAKENHI 23300357 and Japan Science and Technology Agency (JST), A-step feasibility study program (\#AS242Z00112Q).

Received: 17 January 2013 Accepted: 23 May 2013

Published: 31 May 2013

\section{References}

1. Cai Y, Yu X, Hu S, Yu J: A Brief Review on the Mechanisms of miRNA Regulation. Genomics, Proteomics \& Bioinformatics 2009, 7(4):147-154. [http://www.sciencedirect.com/science/article/pii/S1672022908600443]

2. Wienholds E, Kloosterman WP, Miska E, Alvarez-Saavedra E, Berezikov E, de Bruijn E, Horvitz HR, Kauppinen S, Plasterk RH: MicroRNA expression in zebrafish embryonic development. Science 2005, 309(5732):310-311.

3. Wang D, Qiu C, Zhang H, Wang J, Cui Q, Yin Y: Human microRNA oncogenes and tumor suppressors show significantly different biological patterns: from functions to targets. PLOS ONE 2010, 5(9):e13067. [http://dx.doi.org/10.1371]

4. Wittmann J, Jack HM: Serum microRNAs as powerful cancer biomarkers. Biochim Biophys Acta 2010, 1806(2):200-207.

5. Anokye-Danso F, Trivedi CM, Juhr D, Gupta M, Cui Z, Tian Y, Zhang Y, Yang W, Gruber PJ, Epstein JA, Morrisey EE: Highly efficient miRNA-mediated reprogramming of mouse and human somatic cells to pluripotency. Cell Stem Cell 2011, 8(4):376-388.

6. Krichevsky AM, King KS, Donahue CP, Khrapko K, Kosik KS: A microRNA array reveals extensive regulation of microRNAs during brain development. RNA 2003, 9(10):1274-1281.

7. Schratt GM, Tuebing F, Nigh EA, Kane CG, Sabatini ME, Kiebler M, Greenberg ME: A brain-specific microRNA regulates dendritic spine development. Nature 2006, 439(7074):283-289.

8. Miska E, Alvarez-Saavedra E, Townsend M, Yoshii A, Šestan N, Rakic P, Constantine-Paton M, Horvitz H: Microarray analysis of microRNA expression in the developing mammalian brain. Genome Bio/ 2004, 5(9):R68.

9. Shao NY, Hu HY, Yan Z, Xu Y, Hu H, Menzel C, Li N, Chen W, Khaitovich P: Comprehensive survey of human brain micro RNA by deep sequencing. BMC Genomics 2010, 11:409.

10. Shahab S, Matyunina L, Hill C, Wang L, Mezencev R, Walker L, McDonald J: The effects of MicroRNA transfections on global patterns of gene expression in ovarian cancer cells are functionally coordinated. BMC Medical Genomics 2012, 5:33. [http://www.biomedcentral.com/1755-8794/5/33]

11. Shalgi R, Lieber D, Oren M, Pilpel Y: Global and local architecture of the mammalian microRNA-transcription factor regulatory network. PLOS Comput Biol 2007, 3(7):e131.

12. Hobert $O$ : Gene regulation by transcription factors and microRNAs. Science 2008, 319(5871):1785-1786.

13. Hobert $\mathrm{O}$ : Common logic of transcription factor and microRNA action. Trends Biochem Sci 2004, 29(9):462-468

14. Chen K, Rajewsky N: The evolution of gene regulation by transcription factors and microRNAs. Nat Rev Genet 2007, 8(2):93-103.

15. Zhou Y, Ferguson J, Chang JT, Kluger $Y$ : Inter- and intra-combinatorial regulation by transcription factors and microRNAs. BMC Genomics 2007, 8:396. 
16. Cholfin JA, Rubenstein JL: Frontal cortex subdivision patterning is coordinately regulated by Fgf8, Fgf17, and Emx2. J Comp Neurol 2008, 509(2):144-155.

17. Okuno H, Miyashita Y: Expression of the transcription factor Zif268 in the temporal cortex of monkeys during visual paired associate learning. Eur J Neurosci 1996, 8(10):2118-2128.

18. Cheng LE, Zhang J, Reed RR: The transcription factor Zfp423/OAZ is required for cerebellar development and CNS midline patterning. Dev Bio/ 2007, 307:43-52

19. Sun J, Gong X, Purow B, Zhao Z: Uncovering MicroRNA and Transcription Factor Mediated Regulatory Networks in Glioblastoma. PLoS Comput Biol 2012, 8(7):e1002488. [http://dx.doi.org/10.1371]

20. Guo AY, Sun J, Jia P, Zhao Z: A novel microRNA and transcription factor mediated regulatory network in schizophrenia. BMC Syst Biol 2010, 4:10.

21. Taira K: Induction of DNA methylation and gene silencing by short interfering RNAs in human cells. Nature 1176, 441(7097)

22. Mette MF, Aufsatz W, van der Winden J, Matzke MA, Matzke AJ: Transcriptional silencing and promoter methylation triggered by double-stranded RNA. EMBO J 2000, 19(19):5194-5201.

23. Otagaki S, Kawai M, Masuta C, Kanazawa A: Size and positional effects of promoter RNA segments on virus-induced RNA-directed DNA methylation and transcriptional gene silencing. Epigenetics 2011, 6(6):681-691.

24. Halytskiy V: Hypothesis of initiation of DNA methylation de novo and allelic exclusion by small RNAs. Cell and Tissue Bio/ 2008, 2:97-106. [http://dx.doi.org/10.1134/S1990519X08020016]

25. Suzuki MM, Bird A: DNA methylation landscapes: provocative insights from epigenomics. Nat Rev Genet 2008, 9(6):465-476.

26. Palmisano WA, Divine KK, Saccomanno G, Gilliland FD, Baylin SB, Herman JG, Belinsky SA: Predicting lung cancer by detecting aberrant promoter methylation in sputum. Cancer Res 2000, 60(21):5954-5958.

27. Maruyama R, Toyooka S, Toyooka KO, Harada K, Virmani AK, Zochbauer-Muller S, Farinas AJ, Vakar-Lopez F, Minna JD, Sagalowsky A, Czerniak B, Gazdar AF: Aberrant promoter methylation profile of bladder cancer and its relationship to clinicopathological features. Cancer Res 2001, 61(24):8659-8663.

28. Farthing CR, Ficz G, Ng RK, Chan CF, Andrews S, Dean W, Hemberger M, Reik W: Global mapping of DNA methylation in mouse promoters reveals epigenetic reprogramming of Pluripotency genes. PLoS Genet 2008, 4(6):e1000116. [http://dx.plos.org/10.1371]

29. Su Z, Xia J, Zhao Z: Functional complementation between transcriptional methylation regulation and post-transcriptional microRNA regulation in the human genome. BMC Genomics 2011, 12(Suppl 5):S15.

30. Taguchi $Y$ h: Competitive target gene regulation by promoter methylation and miRNA. IPSJ SIG Technical Reports 2012, 2012:1-6. [http://ci.nii.ac.jp/naid/110009459611/en/]

31. Taguchi $Y$ h: Inference of the target gene regulation by miRNA via MiRaGE Server. In Introduction to Genetics ăAş̧ DNA Methylation and Gene Regulation. Edited by Wan J. Hong Kong: iConcept Press; 2013. [in press. http://www.iconceptpress.com/download/paper/12050422170896.pdf]

32. Carter R: The Brain Book: An Illustrated Guide to Its Structure, Function Disorders. London: Dorling Kindersley; 2009.

33. Stuss DT, Floden D: Frontal Cortex. West Sussex: John Wiley \& Sons, Ltd; 2006. [http://dx.doi.org/10.1002/0470018860. s00312]

34. Murray EA: Temporal Cortex. West Sussex: John Wiley \& Sons, Ltd; 2006. [http://dx.doi.org/10.1002/0470018860 s00313]

35. Middleton FA, Tillery SIH: Cerebellum. In Encyclopedia of Cognitive Science. West Sussex: John Wiley \& Sons, Ltd; 2006. [http://dx.doi.org/10.1002/0470018860.s00398]

36. Gibbs JR, van der Brug MP, Hernandez DG, Traynor BJ, Nalls MA, Lai SL, Arepalli S, Dillman A, Rafferty IP, Troncoso J, Johnson R, Zielke HR, Ferrucci L, Longo DL, Cookson MR, Singleton AB: Abundant quantitative trait loci exist for DNA methylation and gene expression in human brain. PLoS Genet 2010, 6(5):e1000952.

37. Yao MJ, Chen G, Zhao PP, Lu MH, Jian J, Liu MF, Yuan XB: Transcriptome analysis of microRNAs in developing cerebral cortex of rat. BMC Genomics 2012, 13:232.

38. Babenko O, Kovalchuk I, Metz GA: Epigenetic programming of neurodegenerative diseases by an adverse environment. Brain Res 2012, 1444:96-111.

39. Roshan R, Ghosh T, Scaria V, Pillai B: MicroRNAs: novel therapeutic targets in neurodegenerative diseases. Drug Discov Today 2009, 14(23-24):1123-1129.

40. Satoh J i, Tabunoki H: Comprehensive analysis of human microRNA target networks. BioData Mining 2011, 4:17. [http://www.biodatamining.org/content/4/1/17]

41. Satoh $\mathrm{J}$ i: Molecular network analysis of human microRNA targetome: from cancers to Alzheimer's disease. BioData Mining 2012, 5:17. [http://www.biodatamining.org/content/5/1/17]

42. Vlachos IS, Kostoulas N, Vergoulis T, Georgakilas G, Reczko M, Maragkakis M, Paraskevopoulou MD, Prionidis K, Dalamagas T, Hatzigeorgiou AG: DIANA miRPath v.2.0: investigating the combinatorial effect of microRNAs in pathways. Nucleic Acids Res 2012, 40(Web Server issue):498-504.

43. Paul A, Cai Y, Atwal GS, Huang ZJ: Developmental coordination of gene expression between synaptic partners during GABAergic circuit assembly in cerebellar cortex. Frontiers in Neural Circuits 2012, 6(37). [http://www. frontiersin.org/neural_circuits/10.3389/fncir.2012.00037/abstract]

doi:10.1186/1756-0381-6-11

Cite this article as: Taguchi: MicroRNA-mediated regulation of target genes in several brain regions is correlated to both microRNA-targeting-specific promoter methylation and differential microRNA expression. BioData Mining 2013 6:11. 